\title{
Numerical Scheme for Solving Stochastic Differential Equations with $G$-Lévy Process
}

\author{
Jiawen Mei, Yifei Xin \\ University of Shanghai for Science and Technology, Shanghai, China \\ Email: 13817612318@163.com
}

How to cite this paper: Mei, J.W. and Xin, Y.F. (2022) Numerical Scheme for Solving Stochastic Differential Equations with $G$-Lévy Process. Journal of Applied Mathematics and Physics, 10, 466-474. https://doi.org/10.4236/jamp.2022.102035

Received: January 23, 2022

Accepted: February 21, 2022

Published: February 24, 2022

Copyright $\odot 2022$ by author(s) and Scientific Research Publishing Inc. This work is licensed under the Creative Commons Attribution International License (CC BY 4.0).

http://creativecommons.org/licenses/by/4.0/

\begin{abstract}
In this paper, we propose numerical schemes for stochastic differential equations driven by $G$-Lévy process under the $G$-expectation framework. By using $G$-Itô formula and $G$-expectation property, we propose Euler scheme and Milstein scheme which have order-1.0 convergence rate. And two numerical experiments including Ornstein-Uhlenbeck and Black-Scholes cases are given.
\end{abstract}

\section{Keywords}

$G$-Lévy Process, $G$-Expectation Property, SDEs, Euler Scheme

\section{Introduction}

The $G$-Brownian motion was introduced by [1] in a sublinear expectation space $(\Omega, \mathcal{H}, \hat{\mathbb{E}})$, which has been applied in finance. Peng also established new sublinear distributions for the $G$-Brownian motion and the related stochastic calculus of Itô type [1] [2] [3]. However, $G$-Brownian motion is considered inadequate to apply in finance because both $G$-Brownian motion and the standard Brownian motion are continuous processes with independent and stationary increments. In this paper, we study the following stochastic differential equation driven by $G$-Lévy process.

$$
\mathrm{d} X_{t}=b\left(t, X_{t}\right) \mathrm{d} t+k\left(t, X_{t}\right) \mathrm{d} \tilde{N}_{t}, \quad t \in[0, T],
$$

where $\tilde{N}_{t}$ is a $G$-Lévy process under the $G$-framework. The operator $b$ is the drift coefficient and $k$ is the jump coefficient.

The research of stochastic differential equations (SDEs) with classical Brownian motion and $G$-Brownian motion has been widely studied [4]. Meanwhile, numerical simulation of $G$-Brownian motion can refer to [5]. Zhou [6] propose a new order-2.0 numerical scheme for Solving Forward-Backward SDEs with Jumps. In [7], Hu focused on the numerical method for solving forward-backward sto- 
chastic differential equations driven by $G$-Brownian motion. Moreover, $\mathrm{Hu}$ also gave an approximate conditional $G$-expectation, which gets methods to calculate the distribution of $G$-Brownian motion. Up to now, almost all the existing works focus on the above Brownian motion.

However, $G$-Brownian motion is considered inadequate to apply in finance because both above Brownian motion is a continuous processes with independent and stationary increments. There is little research concerning stochastic differential equations driven by $G$-Lévy process and its numerical scheme. Therefore, $\mathrm{Hu}$ develop the theory of $G$-Lévy process and its specific case $G$-Poisson process in [8]. And this process is under sublinear expectations with independent and stationary increments but not continuous. In [9], the author concentrated on establishing the integration theory for $G$-Lévy process with finite activity, introduced the Itô formula for general $G$-Itô Lèvy process.

The aim of this paper is to appropriate numerical schemes for solving SDEs driven by $G$-Lévy process under the $G$-expectation framework. We firstly give the $G$-Itô formula and propose Euler scheme to approximate the SDEs with $G$-Lévy process. For comparison purposes, we also gave Milstein Scheme, which also follows from $G$-Itô formula. Then we give two numerical examples including Ornstein-Uhlenbeck and Black-Scholes cases. In particular, we set the function $\lambda(t)$ changes with $t$, applying $G$-expectation property to verify the result that can reach global order-1.0. Moreover, comparative experiments are given through Euler scheme and Milstein Scheme.

The outline of the paper is organized as follows. We introduce some preliminaries such as $G$-Lévy process, its $G$-Itô formula and $G$-expectation in Section 2. In Section 3, we propose Euler scheme and Milstein Scheme for solving the SDEs with $G$-Poisson process. The numerical example is given in Section 4, which is shown to be consistent with the theoretical results.

\section{Preliminaries}

In this section, we will introduce some Preliminaries and notations in the theory of $G$-Lévy process. The $G$-Itô formula is given in this section. More details can be seen in [1] [8]. Let $\Omega$ be a given set and let $\mathcal{H}$ be a vector lattice of real functions defined on $\Omega$, which means if $X \in \mathcal{H}$, then $|X| \in \mathcal{H}$. We will define elements of $\mathcal{H}$ as random variables and there is definition as follow.

Definition 1. (Sublinear expectation) Sublinear expectation $\hat{\mathbb{E}}$ is a functional $\hat{\mathbb{E}}: \mathcal{H} \rightarrow \mathbb{R}$. If all $X, Y \in \mathcal{H}$, there are some properties as follows:

- Monotonicity: If $X \geq Y$ then $\hat{\mathbb{E}}[X] \geq \hat{\mathbb{E}}[Y]$;

- Constant preserving: $\hat{\mathbb{E}}[c]=c$ where all $c \in \mathbb{R}$;

- Sub-additivity: $\hat{\mathbb{E}}[X]-\hat{\mathbb{E}}[Y] \geq \hat{\mathbb{E}}[X-Y]$;

- Positive homogeneity: $\hat{\mathbb{E}}[\lambda X]=\lambda \hat{\mathbb{E}}[X]$ for $\lambda \geq 0$.

Let's define a sublinear expectation space $(\Omega, \mathcal{H}, \hat{\mathbb{E}}),\left(X_{t}\right)_{t \geq 0}$ is called a $d$-dimensional process if $X_{t} \in \mathcal{H}^{d}$ for each $t \geq 0$. And then we will give the definition of $G$-Lévy process under sublinear expectation. 
Definition 2. [2] ( $G$-Lévy process) Assume $X=\left(X_{s}\right)_{s \geq 0}$ defined on a sublinear expectation space $(\Omega, \mathcal{H}, \hat{\mathbb{E}})$ is a Lévy process, $X_{s}^{f}$ is a generalized $G$-Brownian motion and $X_{s}^{g}$ is of finite variation. We define the $X$ as a $G$-Lévy process if the following properties are satisfied:

- for $s \geq 0$, there exists a Lévy process $\left(X_{s}^{f}, X_{s}^{g}\right)$ satisfies $X_{s}=X_{s}^{f}+X_{s}^{g}$.

- $X_{s}^{f}$ and $X_{s}^{g}$ satisfy the following growth conditions:

$$
\lim _{s \downarrow 0} \hat{\mathbb{E}}\left[\left|X_{s}^{f}\right|^{3}\right] s^{-1}=0 ; \quad \hat{\mathbb{E}}\left[\left|X_{s}^{g}\right|\right]<C s \quad \text { for all } s \geq 0,
$$

where $C$ is a positive constant.

Now we will give the $G$-Itô formula.

Lemma 1. [9] ( $G$-Itô formula) For $1 \leq i \leq d, X_{t}^{i}$ is the $k$-th component of $X_{t}$ and it satisfies the following form:

$$
X_{t}^{i}=X_{0}^{i}+\int_{0}^{t} b_{s}^{i} \mathrm{~d} s+\sum_{j=1}^{d} \int_{0}^{t} \sigma_{s}^{i, j} \mathrm{~d} B_{s}^{j}+\int_{0}^{t} \int_{\mathcal{E}} k(e, s) \tilde{N}(\mathrm{~d} e, \mathrm{~d} s),
$$

where $\mathcal{E} \in \mathbb{R}^{q} \backslash\{0\}, B_{s}$ is a $G$-Brownian motion and $\tilde{N}$ (de,ds) is a $G$-Lévy process. For $h \in C_{b}^{2}\left(\mathbb{R}^{q}\right)$, we deduce

$$
\begin{aligned}
& g\left(X_{t}\right)=g\left(X_{0}\right)+\sum_{i=1}^{d} \int_{0}^{t} b_{s}^{i} \frac{\partial g\left(X_{s}\right)}{\partial x_{i}} \mathrm{~d} s+\frac{1}{2} \sum_{i, k=1}^{d} \sum_{j=1}^{d} \int_{0}^{t} \sigma_{s}^{i, j} \sigma_{s}^{k, j} \frac{\partial^{2} g\left(X_{s}\right)}{\partial x_{i} \partial x_{k}} \mathrm{~d}\langle B\rangle_{s} \\
& +\sum_{i=1}^{d} \sum_{j=1}^{d} \int_{0}^{t} \sigma_{s}^{i, j} \frac{\partial g\left(X_{s}\right)}{\partial x_{i}} \mathrm{~d} B_{s}^{j}+\int_{0}^{t} \int_{\mathcal{E}}\left[g\left(X_{s-}+k(e, s)\right)-g\left(X_{s-}\right)\right] \tilde{N}(\mathrm{~d} e, \mathrm{~d} s) .
\end{aligned}
$$

Lemma 2. [7] ( $G$-expectation) For a function $\eta$, we define the approximate $G$-expectation $\hat{\mathbb{E}}_{t_{n}}^{X^{n}}[\eta]$ by

$$
\hat{\mathbb{E}}_{t_{n}}^{X^{n}}[\eta]:=\sup \mathbb{E}_{t_{n}}^{X^{n}}[\eta]
$$

under which $\left(\tilde{N}_{t}\right)_{t \geq 0}$ is the $G$-Lévy process. Similarly, we define the associated discrete sublinear expectation

$$
\hat{\mathbb{E}}^{G}\left[\eta_{t_{n+1}}\right]=\hat{\mathbb{E}}_{t_{0}}^{G, x_{0}}\left[\hat{\mathbb{E}}_{t_{1}}^{G, x_{1}}\left[\cdots \hat{\mathbb{E}}_{t_{n}}^{G, x_{n}}\left[\eta_{t_{n+1}}\right]\right]\right],
$$

\section{Main Results}

The numerical method for the approximate solution of the SDE with $G$-Lévy process is the Euler scheme. Due to Lemma 1, we now introduce the Euler scheme for solving equation.

Scheme 1. (Euler Scheme) [7] For a given partition: $0=t_{0}<\cdots<t_{N-1}<t_{N}=T$ with $\Delta t=\frac{T}{N}$. And we set the initial condition $X_{0}$. For $0 \leq n \leq N-1$, we have

$$
X^{n+1}=X^{n}+b\left(t_{n}, X^{n}\right) \Delta t+k\left(t_{n}, X^{n}\right) \Delta \tilde{N}_{n}
$$

where $\Delta \tilde{N}_{n}=\tilde{N}_{t_{n+1}}-\tilde{N}_{t_{n}}$ is $G$-Lévy process and $\Delta t=t_{n+1}-t_{n}$.

Scheme 2. (Milstein Scheme) [10] For a given partition:

$0=t_{0}<\cdots<t_{N-1}<t_{N}=T$ with $\Delta t=\frac{T}{N}$. And we set the initial condition $X_{0}$.

For $0 \leq n \leq N-1$, we have 


$$
\begin{aligned}
X^{n+1}= & X^{n}+b\left(t_{n}, X^{n}\right) \Delta t+k\left(t_{n}, X^{n}\right) \Delta \tilde{N}_{n} \\
& +\frac{1}{2} k^{2}\left(t_{n}, X^{n}\right)\left(\left(\Delta \tilde{N}_{n}\right)^{2}-\lambda \Delta t-\Delta \tilde{N}_{n}\right) .
\end{aligned}
$$

where $\Delta \tilde{N}_{n}=\tilde{N}_{t_{n+1}}-\tilde{N}_{t_{n}}$ is $G$-Lévy process and $\Delta t=t_{n+1}-t_{n}$.

Remark 1. Thanks to $G$-Itô formula expansion, we can obtain above two weak convergence schemes for solving Equations (1). The first numerical method for solving SDEs with $G$-Lévy process is the Euler scheme, which is the most important and basic method. Thus, we introduce the Euler Scheme for solving SDE with $G$-Lévy process. Then, we also give Milstein Scheme and both Schemes converge to order 1.0. Moreover, in the next section, numerical results are given to verify that our schemes have order-1.0 convergence rate.

\section{Numerical Experiments}

In this section, we consider two one-dimensional SDEs with $G$-Lévy process including Ornstein-Uhlenbeck and Black-Scholes cases to verify the results. Assume terminal time $T=1$, the number of sample paths $N_{s p}=5000$ in the numerical experiment, and the number of time steps is denoted $N \in\left\{2^{3}, 2^{4}, 2^{5}, 2^{6}, 2^{7}\right\}$. We measure the errors of global weak convergence as the following methods:

$$
e_{\Delta t}^{\text {global }}:=\left|\frac{1}{N_{s p}} \sum_{i=1}^{N_{s p}}\left(\varphi\left(X_{i}^{N}\right)-\varphi\left(X_{i, t_{N}}\right)\right)\right|
$$

Therefore, we get the experimental results consistent with the Scheme.

Example 1. Consider the $\mathrm{O}-\mathrm{U}$ process:

$$
\left\{\begin{array}{l}
X_{t}=X_{0}-\int_{0}^{t} b X_{s} \mathrm{~d} s+k \int_{0}^{t} \int_{\mathcal{E}} e \tilde{N}(\mathrm{~d} e, \mathrm{~d} s), \\
X_{0}=1,
\end{array}\right.
$$

where $\tilde{N}(\mathrm{~d} e, \mathrm{~d} s)$ is one-dimensional $G$-poisson process with $\mathcal{E}=[0,1]$ and $\lambda_{\mathcal{E}}=\lambda(t)$. Let $b=-1.5, k=0.01$, and $e \in U(0,1)$, which is Uniform distribution. We apply $G$-Itô formula and $G$-expectation proposition, Equation (6) has the explicit solution

$$
X_{t}=X_{0} \mathrm{e}^{-b t}+k \mathrm{e}^{-b t} \int_{0}^{t} \int_{\mathcal{E}} \mathrm{e}^{b s} e \tilde{N}(\mathrm{~d} e, \mathrm{~d} s) .
$$

In this example, since $\lambda(t)$ is the function of $t$. We utilize the $G$-expectation proposition, taking the maximum $\varphi\left(X^{n}\right), \varphi\left(X_{t_{n}}\right)$ value in $\left[t_{n}, t_{n+1}\right]$. In Order to show the advantages of Scheme 1 in computational efficiency, we also use different coefficients to solve Equation (6). The global errors and convergence rates (CR) of the schemes can be seen in Table 1. We notice that as the time steps increase, the calculated CR approaches 1 . It indicates that we need as large time steps $N$ as possible in numerical experiments, which have stable results. We set $\lambda(t)=t$, and Case 1 with the parameters of $X_{0}=7.5, b=-0.6, k=0.01$, and Case 2 with the parameters of $X_{0}=1, b=-1.5, k=0.01$ in Table 1.

Furthermore, we set $\lambda(t)=t, t^{2}, t^{1 / 2}$. And the left of Figure 1 shows that even if as $\lambda(t)$ changes, the convergence rate of Scheme 1 can still have order-1.0. 

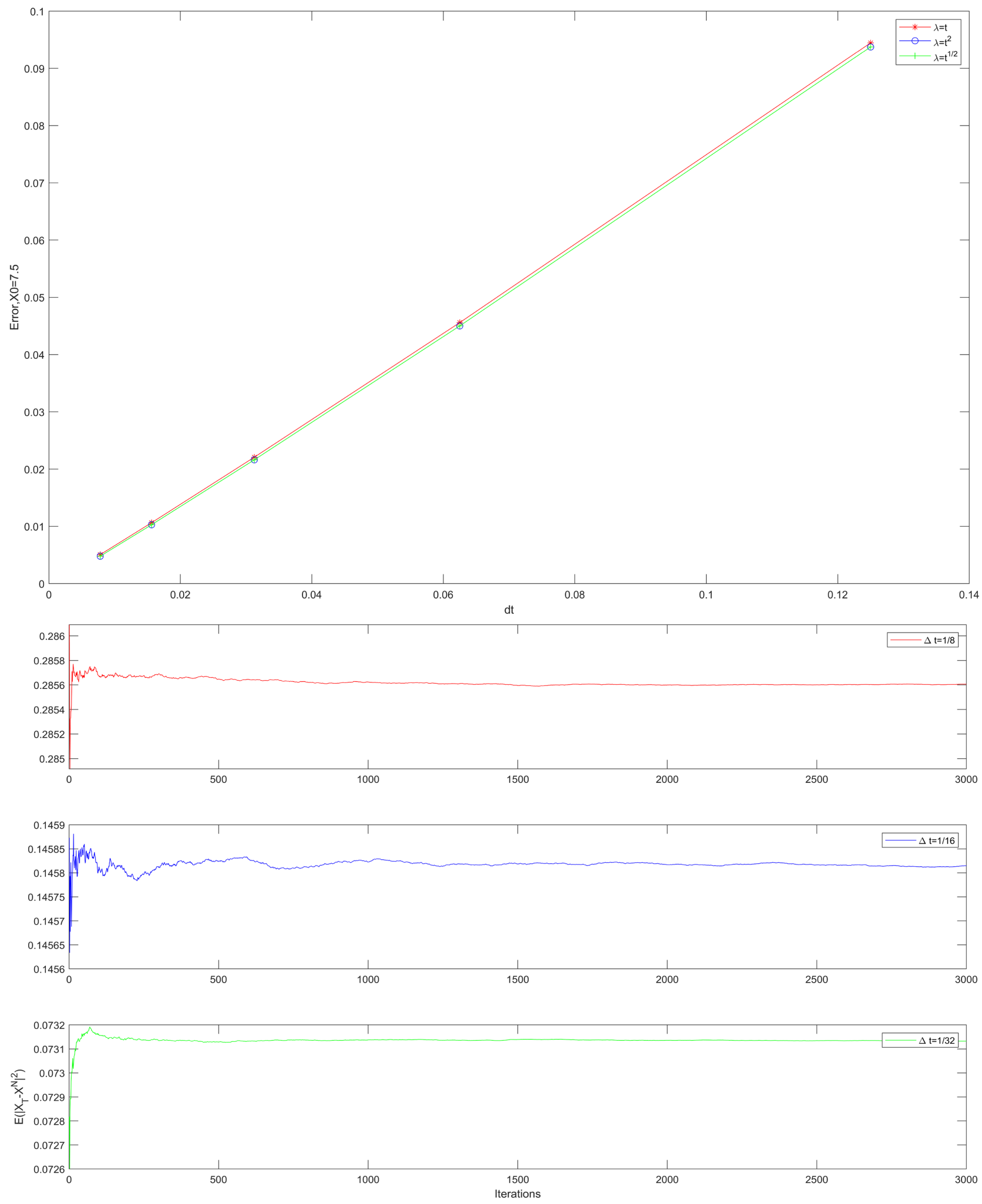

Figure 1. The global errors for Example 1 with different $\lambda(t)$ and the mean-square convergence stability

Then, we testify mean-square convergence stability with $G$-expectation of global errors with the Euler scheme under three kinds of time steps $\Delta t=1 / 8, \Delta t=1 / 16$, 
and $\Delta t=1 / 32$ in the right side of Figure 1.

Example 2. Consider the Black-Scholes model with $G$-pure jump:

$$
\left\{\begin{array}{l}
X_{t}=X_{0}+\int_{0}^{t} b X_{s} \mathrm{~d} s+\int_{0}^{t} \int_{\mathcal{E}} k X_{s} \tilde{N}(\mathrm{~d} e, \mathrm{~d} s), \\
X_{0}=1,
\end{array}\right.
$$

where $\tilde{N}(\mathrm{~d} e, \mathrm{~d} s)$ is $G$-poisson process with $\lambda_{\mathcal{E}}=\lambda(t)$. Let $b=-2, k=0.01$, and $e \in U(0,1)$. We set $\lambda(t)=t, t^{2}, t^{1 / 2}$, applying $G$-Itô formula and $G$-expectation proposition, Equation (6) has the explicit solution:

$$
X_{t}=X_{0} \exp \left\{\int_{0}^{t}(b-\lambda(t) k) \mathrm{d} s+\int_{0}^{t} \int_{\mathcal{E}} \ln (1+k) N(\mathrm{~d} e, \mathrm{~d} s)\right\} .
$$

In this Black-Scholes model, we also take the maximum $\varphi\left(X^{n}\right), \varphi\left(X_{t_{n}}\right)$ value in $\left[t_{n}, t_{n+1}\right]$. In Table 2, we obtain the scheme errors with different coefficients and set $\lambda(t)=t$. Case 1 with the parameters of $b=2, k=0.01$, and Case 2 with the parameters of $b=-2, k=0.01$ in Table 2 .

In addition, we show the change of $\lambda(t)$ of the scheme have the convergence rates of global order-1.0 from the left of Figure 2. On the other hand, from the right of Figure 2, we verify the result of the stability of mean-square convergence under $G$-expectation. The results indicate that Ornstein-Uhlenbeck and Black-Scholes processes show that two examples can achieve order-1.0 convergence under different coefficients. To illustrate the advantages of Scheme in computational efficiency, we also apply Milstein Scheme to solve Equation (7). The convergence rates of Euler Scheme and Milstein Scheme are 1.178 and 1.176 respectively. In particular, for Example 1, its Milstein scheme is Euler scheme. The sample global errors and the corresponding CPU time of the Euler Scheme and Milstein Scheme are displayed in Figure 3. For almost the same order-1.0 of convergence, the Euler scheme takes less CPU time.

Table 1. Errors and CR of Scheme 1 with different coefficients.

\begin{tabular}{ccccc}
\hline$N$ & Case 1 & CR & Case 2 & CR \\
\hline 8 & $2.856 \mathrm{E}-01$ & & $5.193 \mathrm{E}-01$ & \\
16 & $1.416 \mathrm{E}-01$ & 0.9699 & $2.809 \mathrm{E}-01$ & 0.8864 \\
32 & $7.313 \mathrm{E}-02$ & 0.9827 & $1.456 \mathrm{E}-01$ & 0.9174 \\
64 & $3.622 \mathrm{E}-02$ & 0.9932 & $7.350 \mathrm{E}-02$ & 0.9411 \\
128 & $1.774 \mathrm{E}-02$ & 1.0033 & $3.646 \mathrm{E}-02$ & 0.9599 \\
\hline
\end{tabular}

Table 2. Errors and CR of Scheme 1 with different coefficients.

\begin{tabular}{ccccc}
\hline$N$ & Case 1 & CR & Case 2 & CR \\
\hline 8 & $1.385 \mathrm{E}-01$ & & $3.458 \mathrm{E}-02$ & \\
16 & $7.596 \mathrm{E}-01$ & 0.8663 & $1.655 \mathrm{E}-02$ & 1.0679 \\
32 & $3.828 \mathrm{E}-01$ & 0.9274 & $7.771 \mathrm{E}-03$ & 1.0830 \\
64 & $1.744 \mathrm{E}-01$ & 0.9956 & $3.439 \mathrm{E}-03$ & 1.1163 \\
128 & $6.461 \mathrm{E}-02$ & 1.0033 & $1.282 \mathrm{E}-03$ & 1.1781 \\
\hline
\end{tabular}



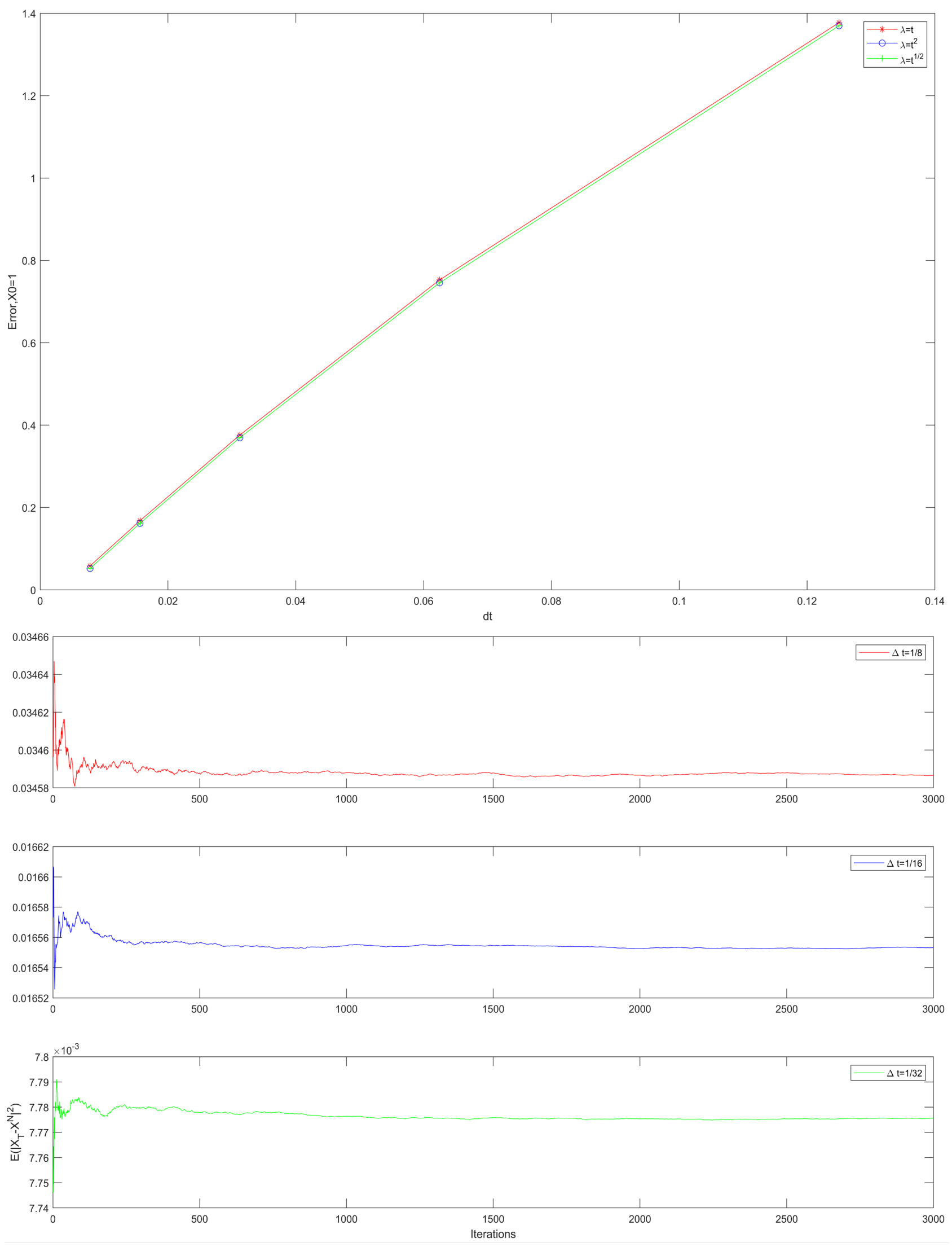

Figure 2. The global errors for Example 2 with different $\lambda(t)$ and the mean-square convergence stability. 


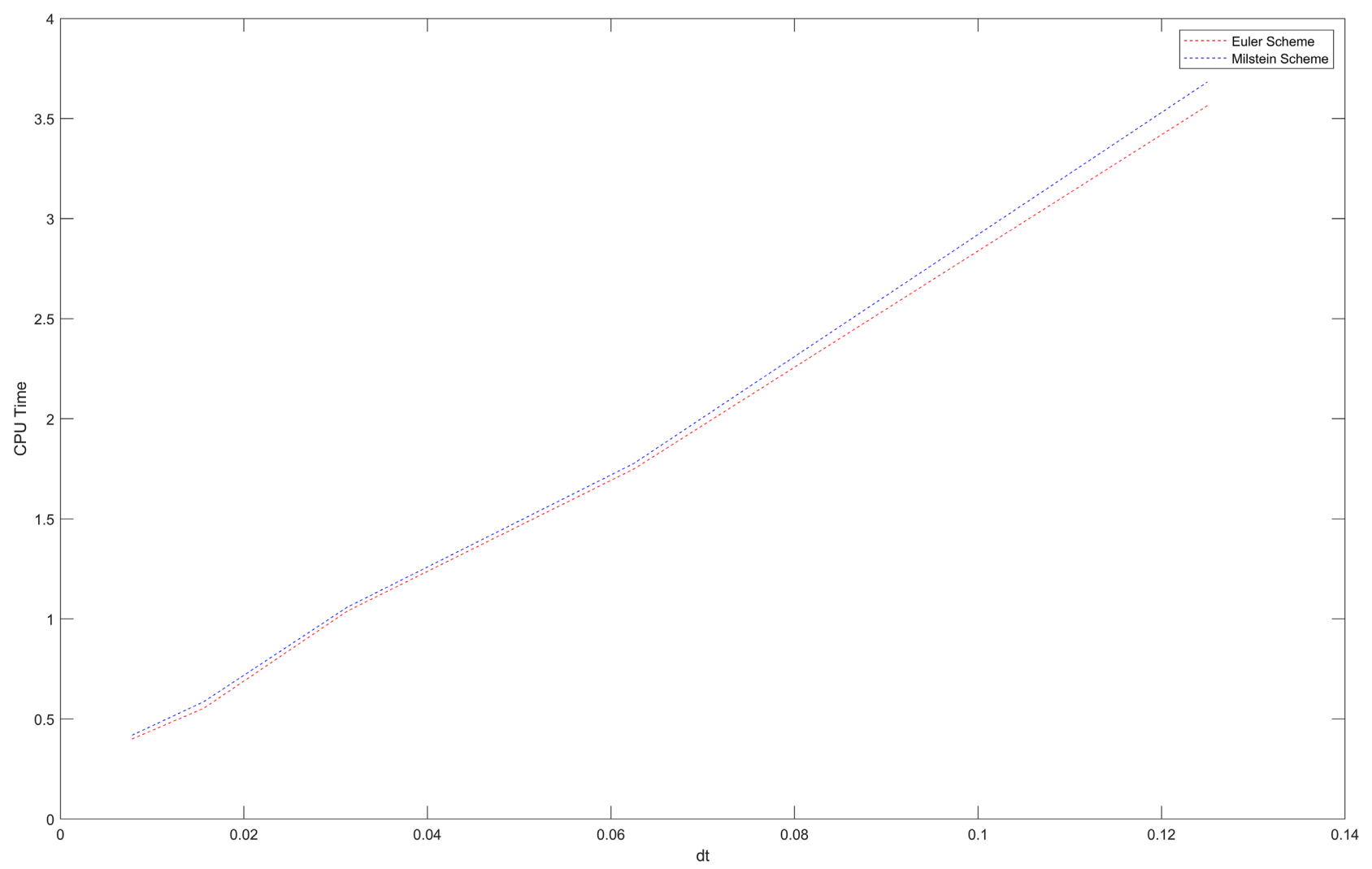

Figure 3. The CPU Time for Example 2 with $d t$.

\section{Conclusion}

In this paper, we propose Euler scheme for solving stochastic differential equations with $G$-Lévy process. In numerical experiments, we used two examples including Ornstein-Uhlenbeck and Black-Scholes processes to verify that the convergence of scheme is order-1.0. Furthermore, we compared the different coefficients and the change of $\lambda(t)$, applying $G$-Itô formula and $G$-expectation proposition, and got that the scheme has order-1.0.

\section{Conflicts of Interest}

The authors declare no conflicts of interest regarding the publication of this paper.

\section{References}

[1] Peng, S. (2007) $G$-Expectation, $G$-Brownian Motion and Related Stochastic Calculus of Itô Type. In: Benth, F.E., Di Nunno, G., Lindstrøm, T., Øksendal, B. and Zhang, T., Eds., Stochastic Analysis and Applications, Abel Symposia, Vol 2., Springer, Berlin, Heidelberg, 541-567. https://doi.org/10.1007/978-3-540-70847-6_25

[2] Peng, S. (2007) G-Brownian Motion and Dynamic Risk Measure under Volatility Uncertainty.

[3] Peng, S. (2008) Multi-Dimensional G-Brownian Motion and Related Stochastic Calculus under G-Expectation. Stochastic Processes and Their Applications, 118, 2223-2253. https://doi.org/10.1016/j.spa.2007.10.015 
[4] Platen, E. and Bruti-Liberati, N. (2010) Numerical Solution of Stochastic Differential Equations with Jumps in Finance. Springer Science.

https://doi.org/10.1007/978-3-642-13694-8

[5] Yang, J. and Zhao, W. (2016) Numerical Simulations for G-Brownian Motion. Frontiers of Mathematics in China, 11, 1625-1643.

https://doi.org/10.1007/s11464-016-0504-9

[6] Zhou, H., Li, Y. and Wang, Z. (2016) A New Second Order Numerical Scheme for Solving Forward Backward Stochastic Differential Equations with Jumps. Applied Mathematics, 7, 1408-1414. https://doi.org/10.4236/am.2016.712121

[7] Hu, M. (2021) An Efficient Numerical Method for Forward-Backward Stochastic Differential Equations Driven by $G$-Brownian Motion. Applied Numerical Mathematics, 165, 578-597. https://doi.org/10.1016/j.apnum.2021.03.012

[8] Hu, M. and Peng, S. (2009) G-Lévy Processes under Sublinear Expectations.

[9] Krzysztof, P. (2012) Itô Calculus and Jump Diffusions for $G$-Lévy Processes.

[10] Ren, Q. and Tian, H. (2020) Compensated $\theta$-Milstein Methods for Stochastic Differential Equations with Poisson Jumps. Applied Numerical Mathematics, 150, 27-37. https://doi.org/10.1016/j.apnum.2019.09.009 